\title{
Hyperbolic Center of Mass for a System of Particles in a Two-Dimensional Space with Constant Negative Curvature: An Application to the Curved 2-Body Problem
}

\author{
Pedro Pablo Ortega Palencia ${ }^{1}$ (D), Ruben Dario Ortiz Ortiz ${ }^{2 *}$ (D) and Ana Magnolia Marin Ramirez ${ }^{2}$ (D) \\ 1 Grupo Ecuaciones Diferenciales, Universidad de Cartagena, Cartagena de Indias 130014, Colombia; \\ portegap@unicartagena.edu.co \\ 2 Grupo Ondas, Universidad de Cartagena, Cartagena de Indias 130014, Colombia; \\ amarinr@unicartagena.edu.co \\ * Correspondence: rortizo@unicartagena.edu.co
}

check for updates

Citation: Ortega Palencia, P.P.; Ortiz Ortiz, R.D.; Marín Ramírez, A.M. Hyperbolic Center of Mass for a System of Particles in a

Two-Dimensional Space with Constant Negative Curvature: An Application to the Curved 2-Body Problem. Mathematics 2021, 9, 531. https://doi.org/10.3390/math9050531

Academic Editor: Dumitru Baleanu

Received: 17 December 2020

Accepted: 27 February 2021

Published: 3 March 2021

Publisher's Note: MDPI stays neutral with regard to jurisdictional claims in published maps and institutional affiliations.

Copyright: (c) 2021 by the authors. Licensee MDPI, Basel, Switzerland. This article is an open access article distributed under the terms and conditions of the Creative Commons Attribution (CC BY) license (https:// creativecommons.org/licenses/by/ $4.0 /)$.

\begin{abstract}
In this article, a simple expression for the center of mass of a system of material points in a two-dimensional surface of Gaussian constant negative curvature is given. By using the basic techniques of geometry, we obtained an expression in intrinsic coordinates, and we showed how this extends the definition for the Euclidean case. The argument is constructive and serves to define the center of mass of a system of particles on the one-dimensional hyperbolic sphere $\mathbb{L}_{R}^{1}$.
\end{abstract}

Keywords: center of mass; conformal metric; geodesic; hyperbolic lever law

\section{Introduction}

The center of mass (center of gravity or centroid) is a fundamental concept, and its geometrical and mechanical properties are for understanding many physical phenomena. Its definition for Euclidean spaces is elemental; nevertheless, in spaces with the non-zero curvature, it is rare. In [1], the author gives an extensive explanation showing that the possibility of the concept can be correctly defined in more general spaces, and he signalizes the difficulties in defining spaces of non-zero curvature concerning the lack of the linear structure of ones. While it is true that the author synthesizes the basic properties of the center of mass, in his approach there are some entities without physical meaning, such as the non-conservation of the total mass of the system or the presence of unbounded speeds under normal conditions. In [2], there is a definition of center of mass for two particles in hyperbolic space, in the same direction to the one presented here, but the authors do not give an expression for calculating it. In [3], the author mentions the difficulty of defining the center of mass in curved spaces. He provides a class of orbits in the curved $n$-body problem for which "no point that could play the role of the center of mass is fixed or moves uniformly along a geodesic". This proves that the equations of motion lack center-of-mass and linear momentum integrals. Nevertheless, he does not provide a way to calculate or determine this element. In [4], the center of the mass problem on two-point homogeneous spaces and the connection of existing mass center concepts with the two-body Hamiltonian functions are considered. We discussed different possibilities for defining a center of the mass in spaces of constant and non-zero curvature, and it was established that a natural way of defining a concept of center of mass for two particles on a Riemannian space as the point on the shortest geodesic interval joining these particles that divides the interval in the ratio of the masses of particles and this is denoted by $R_{1}$.

This last approach is followed in the present work.

In this article, the problem of finding a mathematical expression for computing the center of mass of a system of $n$ particles sited on the two-dimensional hyperbolic sphere $\mathbb{L}_{R}^{2}=\left\{(x, y, z): x^{2}+y^{2}-z^{2}=-R^{2}\right\}$ is considered. The stereographic projection of the 
upper sheet of $\mathbb{L}_{R}^{2}$ on the Poincaré disk $\mathbb{D}_{R}^{2}=\left\{(x, y): x^{2}+y^{2}<R^{2}\right\}=\{w \in \mathbb{C}:|w|<R\}$, endowed with the conformal metric (see [5]).

$$
d s^{2}=\frac{4 R^{4} d w d \bar{w}}{\left(R^{2}-|w|^{2}\right)^{2}}
$$

Both $\mathbb{D}_{R}^{2}$ with the metric (1) and $\mathbb{L}_{R}^{2}$ with the Euclidean metric have the same Gaussian curvature $k=-1 / R^{2}$, and for the Minding's Theorem they belong to the isometric differentiable class (see [6,7], chapter 2). In [8], we use the lever law, an explicit formula that allows us to calculate the center of mass of a system of $n$ particles with masses $m_{1}, m_{2}, \ldots, m_{n}>0$, located on the superior half plane of Lobachevsky $H^{2}$, endowed with a conformal metric which induces a constant and negative Gaussian curvature.

Following the basic geometry methods, we obtain the expression for the center of mass for a system of $n$ particles sited in the hyperbolic sphere $\mathbb{L}_{R}^{2}$ with arbitrary $R$.

We organized this article as follows: In Section 1 we introduced some concepts relative to the center of mass in the Euclidean spaces. In Section 2, some properties of stereographical projection are remembered, and we proceeded to deduce the expression for the center of mass, for two particles on the upper branch of hyperbola, from the "hyperbolic rule of the lever" (see $[1,4]$ ) extended to the surface of $\mathbb{L}_{R}^{2}$. After obtaining the expression for the center of mass for two particles in $\mathbb{L}_{R}^{1}$, we naturally extended to a system of $n$ particles in $\mathbb{L}_{R}^{1}$, and in the same way, to a system of $n$ particles in $\mathbb{L}_{R}^{2}$.

\section{One-Dimensional Euclidean Case}

Let us consider two particles with positive masses sited in the real line at points $x_{1}$ and $x_{2}$. The point defines the center of mass of the system

$$
x_{c}=\frac{m_{1} x_{1}+m_{2} x_{2}}{m_{1}+m_{2}}
$$

A direct calculation shows that $m_{1}\left|x_{c}-x_{1}\right|=m_{2}\left|x_{c}-x_{2}\right|$ (Euclidean rule of the lever). It is easy to prove that $x_{c}$ is the unique point in a segment (geodesic) joining $x_{1}$ and $x_{2}$ with this property. We extended this definition to more dimensions in Euclidean spaces. Nevertheless, this definition cannot be extended to spaces in general because it is possible in such cases that it is not defined as a linear structure. However, with the "rule of the lever" in mind is possible to have this definition to Riemannian surfaces, as we shall see later.

\section{Center of Masses in a Two-Dimensional Hyperbolic Space}

3.1. Some Observations about the Stereographic Projection of Hyperbolic Sphere on the Poincaré Disk

Let $\mathbb{D}_{R}^{2}=\{w \in \mathbb{C}:|w|<R\}$ and $P: \mathbb{L}_{R}^{2} \rightarrow \mathbb{D}_{R}^{2}$ be the stereographic projection; then, for $(x, y, z) \in \mathbb{L}_{R}^{2}$, we have $P(x, y, z)=w=u+i v$, where $u=\frac{R x}{R+z}, v=\frac{R y}{R+z}$ and the inverse projection is $P^{-1}: \mathbb{D}_{R}^{2} \rightarrow \mathbb{L}_{R}^{2}$, where (see [5]),

$$
P^{-1}(u+i v)=\left(\frac{2 R^{2} u}{R^{2}-u^{2}-v^{2}}, \frac{2 R^{2} v}{R^{2}-u^{2}-v^{2}}, \frac{R\left(u^{2}+v^{2}+R^{2}\right)}{R^{2}-u^{2}-v^{2}}\right) .
$$

$P^{-1}$ transforms lines through the origin in meridians (hyperbolas through the point $(0,0, R)$, with the axis being the $z$-axis) and circles with center at the origin, $\left\{w \in \mathbb{D}_{R}^{2}:|w|=\right.$ const. $<R\}$ in parallels (horizontal concentric circles on the upper sheet of hyperboloid).

If we consider the stereographic projection of the one-dimensional hyperbolic sphere $\mathbb{L}_{R}^{1}$ on the real line, we reduce the above equation to

$P(x, y)=u$ where $u=\frac{R x}{R+y}, u \in(-R, R)$, and the inverse projection is

$$
P^{-1}(u)=\left(\frac{2 R^{2} u}{R^{2}-u^{2}}, \frac{R\left(R^{2}+u^{2}\right)}{R^{2}-u^{2}}\right) .
$$


Theorem 1. Let us consider two masses $m_{1}, m_{2}$ sited at the points $Q_{1}, Q_{2}$, respectively, and let $Q_{c}\left(x_{c}, y_{c}\right)$ be the coordinates of the hyperbolic center of mass, and $s_{1}$ the length of the arc from $Q_{1}$ to $Q_{c}$ and $s_{2}$ the length of the arc from $Q_{c}$ to $Q_{2}$; then, from the relation (hyperbolic rule of the lever) $m_{1} r_{1}=m_{2} r_{2}$ it follows that:

$$
\left(\frac{R+u_{c}}{R-u_{c}}\right)^{m}=\left(\frac{R+u_{1}}{R-u_{1}}\right)^{m_{1}}\left(\frac{R+u_{2}}{R-u_{2}}\right)^{m_{2}} .
$$

where $m=m_{1}+m_{2}$ is the total mass of the system.

Proof. In this case, the length of the arc from the South Pole $P_{s}$ to the arbitrary point $(x, y)$ is

$$
r=\int_{0}^{u} \frac{2 R^{2} d t}{R^{2}-t^{2}}=R \ln \left(\frac{R+u}{R-u}\right) .
$$

More generally, the length of the arc $s$ from point $Q_{1}\left(x_{1}, y_{1}\right)$ to $Q_{2}\left(x_{2}, y_{2}\right)$ in the same parallel, if their stereographical projections are $u_{1}$ and $u_{2}$, is

$$
r=R\left(\ln \left(\frac{R+u_{2}}{R-u_{2}}\right)-\ln \left(\frac{R+u_{1}}{R-u_{1}}\right)\right) .
$$

Thus,

$$
r_{1}=R\left(\ln \left(\frac{R+u_{1}}{R-u_{1}}\right)-\ln \left(\frac{R+u_{c}}{R-u_{c}}\right)\right)
$$

and

$$
\begin{gathered}
r_{2}=R\left(\ln \left(\frac{R+u_{c}}{R-u_{c}}\right)-\ln \left(\frac{R+u_{2}}{R-u_{2}}\right)\right), \\
R m_{1}\left(\ln \left(\frac{R+u_{c}}{R-u_{c}}\right)-\ln \left(\frac{R+u_{1}}{R-u_{1}}\right)\right)=R m_{2}\left(\ln \left(\frac{R+u_{2}}{R-u_{2}}\right)-\ln \left(\frac{R+u_{c}}{R-u_{c}}\right)\right) .
\end{gathered}
$$

Therefore,

$$
\ln \left(\frac{R+u_{c}}{R-u_{c}}\right)=\frac{1}{m_{1}+m_{2}}\left(m_{1} \ln \left(\frac{R+u_{1}}{R-u_{1}}\right)+m_{2} \ln \left(\frac{R+u_{2}}{R-u_{2}}\right)\right) .
$$

and from there the result follows.

Inductively, we can extend the last argument to $n$ particles with masses $m_{1}, m_{2}, \ldots, m_{n}$ sited on $\mathbb{L}_{R}^{1}$ as expressed by the following.

Corollary 1. Let $m_{1}, m_{2}, \ldots, m_{n}$ be positive masses sited on the points $\left(x_{1}, y_{1}\right),\left(x_{2}, y_{2}\right), \ldots,\left(x_{n}, y_{n}\right)$ of $\mathbb{L}_{R}^{1}$ with stereographical projections $u_{1}, u_{2}, \ldots, u_{n}$, respectively. Then there is a unique point $u_{c} \in(-R, R)$ such that such that the following expression is fulfilled:

$$
\left(\frac{R+u_{c}}{R-u_{c}}\right)^{m}=\prod_{k=1}^{n}\left(\frac{R+u_{k}}{R-u_{k}}\right)^{m_{k}}
$$

where $m=\sum_{k=1}^{n} m_{k}$ is the total mass of the system.

\subsection{Center of Mass for a System of Two Particles in $\mathbb{L}_{R}^{2}$}

Now, we extend the "rule of the lever" to a more general context: 
Consider a Riemannian surface $T$ and two particles with masses $m_{1}, m_{2}$ sited in the points $x_{1}, x_{2} \in T$, respectively. Then we define the $T$-center of mass as the point $x_{c}$ in the geodesic joining $x_{1}$ to $x_{2}$ such that the following relation is verified:

$$
m_{1} d\left(x_{1}, x_{c}\right)=m_{2} d\left(x_{2}, x_{c}\right)
$$

where $d$ is the metric in $T$, and $d\left(x_{1}, x_{c}\right)+d\left(x_{c}, x_{2}\right)=d\left(x_{1}, x_{2}\right)$. For the case of $\mathbb{L}_{R}^{2}$, geodesics are hyperbolas determined for the intersection of the upper sheet of the hyperboloid with the plane drawn for the pair of points and the origin $(0,0,0)$.

Now we can calculate the hyperbolic center of mass for a system with a finite number of particles on $\mathbb{L}_{R}^{2}$. Following exactly the same reasoning as in the previous section, we obtain the following.

Corollary 2. Let $m_{1}, m_{2}, \ldots, m_{n}$ be $n$ masses of particles sited, respectively, in the points $\left(x_{1}, y_{1}, z_{1}\right)$, $\left(x_{2}, y_{2}, z_{2}\right), \ldots,\left(x_{n}, y_{n}, z_{n}\right)$ on the same geodesic of $\mathbb{L}_{R}^{2}$ with stereographical projections $w_{1}, w_{2}$, $\ldots, w_{n}$, in the Poincaré disk. And let $w_{c}$ be their hyperbolic center of mass; then, the next relation is fulfilled:

$$
\left(\frac{R+w_{c}}{R-w_{c}}\right)^{m}=\prod_{k=1}^{n} m_{k}\left(\frac{R+w_{k}}{R-w_{k}}\right)^{m_{k}}
$$

where $m=\sum_{k=1}^{n} m_{k}$.

Remark 1. If each fraction is divided, their numerator and denominator for $R$ and both sides rise to the power $R$, when $R \rightarrow \infty$, is obtained,

$$
\exp \left(2 m w_{c}\right)=\exp \left(2 \sum_{k=1}^{n} m_{k} w_{k}\right)
$$

Or equivalently,

$$
w_{c}=\frac{1}{m} \sum_{k=1}^{n} m_{k} w_{k}
$$

This corresponds to the equation for the center of mass in the Euclidean complex plane, that is, the complex plane (or $\mathbb{R}^{2}$ ), with Euclidean metric and zero curvature.

\section{An Application to the Curved 2-Body Problem}

In [5], the curved $n$-body problem in a two-dimensional space with constant negative curvature is studied, and the model $\mathbb{L}_{R}^{2}$ is considered, in which there are systems. Let $z=\left(z_{1}, z_{2}, \ldots, z_{n}\right) \in\left(D_{R}^{2}\right)^{n}$ be the configuration of $n$ point particles with masses $m_{1}, m_{2}, \ldots, m_{n}>0 \in \mathbb{D}_{R}^{2}$

$$
m_{k} \ddot{z}_{k}=-\frac{2 m_{k} \bar{z}_{k} \dot{z}_{k}^{2}}{R^{2}-\left|z_{k}\right|^{2}}+\frac{2}{\lambda\left(z_{k}, \bar{z}_{k}\right)} \frac{\partial U_{R}}{\partial \bar{z}_{k}}, \quad k=1, \ldots, n
$$

where

$$
\lambda\left(z_{k}, \bar{z}_{k}\right)=\frac{4 R^{4}}{\left(R^{2}-\left|z_{k}^{2}\right|\right)^{2}}
$$

is the conformal function of the Riemannian metric,

$$
\begin{gathered}
\frac{\partial U_{R}}{\partial \bar{z}_{k}}=\sum_{j=1, j \neq k}^{n} \frac{2 m_{k} m_{j} R P_{2,(k, j)}\left(z, \bar{z}_{k}\right)}{\left(\Theta_{2,(k, j)}\left(z, \bar{z}_{k}\right)\right)^{3 / 2}}, \\
P_{2,(k, j)}\left(z, \bar{z}_{k}\right)=\left(R^{2}-\left|z_{k}\right|^{2}\right)\left(R^{2}-\left|z_{j}\right|^{2}\right)^{2}\left(z_{j}-z_{k}\right)\left(R^{2}-z_{k} \bar{z}_{j}\right),
\end{gathered}
$$




$$
\begin{gathered}
\Theta_{2,(k, j)}\left(z, \bar{z}_{k}\right)=\left[2\left(z_{k} \bar{z}_{j}+z_{j} \bar{z}_{k}\right) R^{2}-\left(\left|z_{k}\right|^{2}+R^{2}\right)\left(\left|z_{j}\right|^{2}+R^{2}\right)\right]^{2}-\left(R^{2}-\left|z_{k}\right|^{2}\right)^{2}\left(R^{2}-\left|z_{j}\right|^{2}\right)^{2}, \\
k, j \in\{1, \ldots, n\}, k \neq j .
\end{gathered}
$$

We consider functions of the form

$$
w_{k}(t)=e^{i t} z_{k}(t),
$$

where $z=\left(z_{1}, \ldots, z_{n}\right)$ is a solution of Equation (10). Straightforward computations show that

$$
\left\{\begin{array}{l}
\dot{w}_{k}=\left(i z_{k}+\dot{z}_{k}\right) e^{i t} \\
\ddot{w}_{k}=\left(\ddot{z}_{k}+2 i \dot{z}_{k}-z_{k}\right) e^{i t} \\
\frac{d \bar{z}_{k}}{d \bar{w}_{k}}=e^{i t}, \quad k=1, \ldots, n .
\end{array}\right.
$$

For the configurations called relative equilibrium, concerning the 2-body problem, the next result is established.

Theorem 2. Consider two point particles of masses $m_{1}, m_{2}>0$ moving on the Poincare disk $\mathbb{D}_{R^{\prime}}^{2}$ whose center is the origin, 0 , of the coordinate system. Then $z=\left(z_{1}, z_{2}\right)$ is an elliptic relative equilibrium of system (10) with $n=2$ if and only if, for every circle centered at 0 of radius $\alpha$, with $0<\alpha<R$, along which $m_{1}$ moves, there is a unique circle centered at 0 of radius $r$, which satisfies $0<r<R$, along which $m_{2}$ moves, such that, at every time instant, $m_{1}$ and $m_{2}$ are on some diameter of $\mathbb{D}_{R}^{2}$, with 0 between them. Moreover,

1. if $m_{2}>m_{1}>0$ and $\alpha$ are given, then $r<\alpha$;

2. if $m_{1}=m_{2}>0$ and $\alpha$ are given, then $r=\alpha$;

3. if $m_{1}>m_{2}>0$ and $\alpha$ are given, then $r>\alpha$.

This result was reformulated in a more precise form, using the expression for the hyperbolic center of mass taking into account that in a configuration corresponding to a relative equilibrium is invariant with the time, because the distance and angles between particles do not change. This is sufficient, considering the initial configuration on the $x$-axis, and $\alpha$ corresponds to the length measure over the Poincaré disk of the projection of arc $r_{1}$ over the hyperbolic sphere $\mathbb{L}_{R}^{2}$, and $r$ is the projection length in disk one of the arc $r_{2}$ over the hyperbolic sphere. Then we have the next relations:

$$
r_{1}=\ln \left(\frac{R+\alpha}{R-\alpha}\right)
$$

and

$$
r_{2}=-\ln \left(\frac{R+r}{R-r}\right) .
$$

Substituting in the hyperbolic rule $m_{1} r_{1}=m_{2} r_{2}$ and the expression for the center of mass, we obtain

$$
\left(\frac{R+\alpha}{R-\alpha}\right)^{m_{1}}=\left(\frac{R+r}{R-r}\right)^{-m_{2}} .
$$

Thus it follows from Equation (6) that

$$
\frac{R-w_{c}}{R+w_{c}}=1
$$

It follows that $w_{c}=0$ and so the center of mass is fixed for every time in the South Pole of the hyperbolic sphere $(0,0, R)$, and Theorem 2 can be expressed in the following form. 
Theorem 3. For every configuration of elliptic relative equilibrium for the 2-body problem with masses $m_{1}, m_{2}$ sited in the points $P_{1}\left(x_{1}, y_{1}, z_{1}\right)$ and $P_{2}\left(x_{2}, y_{2}, z_{2}\right)$ on the hyperbolic sphere of radius $R$. If $r_{1}$ and $r_{2}$ are the lengths of arcs measured from the South Pole $(0,0, R)$ to the points $P_{1}$ and $P_{2}$, respectively, then it satisfies the relation $m_{1} r_{1}=m_{2} r_{2}$, and the center of mass of the system is fixed in $(0,0, R)$ for every time.

The hyperbolic spaces are very special in relativity. A hyperbolic (i.e., Lobachevskian) space can be represented upon one sheet of a two-sheeted cylindrical hyperboloid in Minkowski space-time. According to works that recently appeared in literature (see [9]), in hyperbolic spaces, the expression for the center of mass obtained by adopting the relativistic rule of lever reads

$$
m_{1} \sinh \sqrt{-k} r_{1}=m_{2} \sinh \sqrt{-k} r_{2},
$$

with $r_{i}, i=1,2$, denoting the Riemannian distance of $m_{i}$ to the center of mass and $k$ the (negative) Gaussian curvature, respectively. Using the stereographic projection of a hyperbolic sphere on the Poincaré disk.

For both the Euclidean and the hyperbolic spaces, the center of mass for the system particles plays a central role in the conserved momentum principle. Adoption of the conserved momentum principle for 2-body is expressed in spaces with negative Gaussian curvature is along the following lines.

Theorem 4. Consider two masses $m_{1}, m_{2}$ sited in the points $Q_{1}, Q_{2}$, respectively, and $r_{1}, r_{2}$ the length or arc from $Q_{c}$ to $Q_{2}$; then, from the relation (hyperbolic rule of the lever) $m_{1} \sinh \sqrt{-k} r_{1}=$ $m_{2} \sinh \sqrt{-k} r_{2}$ and using the stereographic projection of a hyperbolic sphere on the Poincare disk the conserved momentum principle for 2-body expressed in spaces with negative Gaussian curvature is

$$
m_{1} \sinh \left(R \ln \left(\frac{R+w_{c}}{R-w_{c}} \frac{R-w_{1}}{R+w_{1}}\right)\right)=m_{2} \sinh \left(R \ln \left(\frac{R-w_{c}}{R+w_{c}} \frac{R+w_{2}}{R-w_{2}}\right)\right)
$$

Proof. Following the ideas from [1] on relativistic momentum, we have

$$
p=m \frac{v}{\sqrt{1-v^{2}}}
$$

where $p$ is the momentum, $v$ is the velocity of the particle with respect to a frame of reference and the velocity of the light is $c=1$. We take

$$
r=\frac{1}{2} \ln \left(\frac{1+v}{1-v}\right)
$$

the distance of the particle with respect to the center of the reference frame. This solution with respect to $v$ yields

$$
v=\tanh r .
$$

Evaluating this in the momentum gives $p=m \sinh r$. From the energy of the particle $E=m \frac{1}{\sqrt{1-v^{2}}}$ and replacing the velocity of the particle $v=\tanh r$ gets $E=m \cosh r$. From the hyperbolic identity $\cosh x^{2}-\sinh x^{2}=1$, the constant $E^{2}-p^{2}=m^{2}$ can be obtained. Let $r_{1}$ be the distance between the particle with mass $m_{1}$ and the mass center, and $r_{2}$ the distance between the particle with mass $m_{2}$ and the mass center; then, $v_{1}=$ $\tanh r_{1}$, and $v_{1}=\tanh r_{1}$ are the velocities of particles with mass $m_{1}$ and $m_{2}$, respectively. In consequence, the relativistic momentum is constant,

$$
m_{1} \sinh r_{1}=m_{2} \sinh r_{2}
$$


The expression of the center of mass for a system of two particles in $L_{\mathbb{R}}^{2}$ is presented in the following result.

Theorem 5. Consider two masses $m_{1}, m_{2}$ sited in the points $Q_{1}, Q_{2}$, respectively, and $r_{1}, r_{2}$ the length or arc from $Q_{c}$ to $Q_{2}$; then, from the relation (hyperbolyc rule of the lever) $m_{1} \sinh \sqrt{-k} r_{1}=$ $m_{2} \sinh \sqrt{-k} r_{2}$ and using the stereographic projection of a hyperbolic sphere on the Poincare disk, the center of mass for a system of two particles in $L_{\mathbb{R}}^{2}$ is given by

$$
m=m_{1} \cosh \left(\sqrt{-k} \ln \left(\frac{\left(R+w_{c}\right)\left(R-w_{1}\right)}{\left(R-w_{c}\right)\left(R+w_{1}\right)}\right)\right)+m_{2} \cosh \left(\sqrt{-k} \ln \left(\frac{\left(R-w_{\mathcal{c}}\right)}{\left(R+w_{c}\right)} \frac{\left(R+w_{2}\right)}{\left(R-w_{2}\right)}\right)\right)
$$

where $k$ is the (negative) Gaussian curvature

Proof. From the principle of conservation of relativistic momentum and total energy we obtain the desired result. Following the ideas from [1],

$$
E=m=m_{1} \cosh r_{1}+m_{2} \cosh r_{2}
$$

with $E=m \cosh 0=m$.

One of the most interesting aspects concerning the determination of the center of mass of a particle system lies in its physical applications. As known, for Euclidean space, Equations (2) may be derived from the Lever rule. If we suppose that the particles are under the influence of an attractive potential force, depending only on their mutual distance, this equation may be derived from the other two different characteristics of the center of mass: (a) Collision point and (b) center of steady rotation.

In situation (a), for a collisional point, if the particles are initially at rest they will collide at the center of mass; the expression of the centre of mass is along the following lines.

Theorem 6. Consider two masses $m_{1}, m_{2}$ sited in the points $Q_{1}, Q_{2}$, respectively, and $r_{1}, r_{2}$ the length or arc from $Q_{c}$ to $Q_{2}$; then, from the relation (hyperbolyc rule of the lever) $m_{1} r_{1}=m_{2} r_{2}$ and using the stereographic projection of a hyperbolic sphere on the Poincare disk the center of mass for a system of two particles in $L_{\mathbb{R}}^{2}$ is given by

$$
\left(\frac{1+\sqrt{-k} w_{c}}{1-\sqrt{-k} w_{c}}\right)^{m}=\left(\frac{1+\sqrt{-k} w_{1}}{1-\sqrt{-k} w_{1}}\right)^{m_{1}}\left(\frac{1+\sqrt{-k} w_{2}}{1-\sqrt{-k} w_{2}}\right)^{m_{2}} .
$$

Proof. Following the ideas from [9] we obtain our result.

In situation (b), for the center of steady rotation, if the particles rotate uniformly along with concentric circles, maintaining a constant distance over time, then the center of mass coincides with the circle's center, the expression of the center of mass is given by the following.

Theorem 7. Consider two masses $m_{1}, m_{2}$ sited in the points $Q_{1}, Q_{2}$, respectively, and $r_{1}, r_{2}$ the length or arc from $Q_{c}$ to $Q_{2}$; then, from the relation (hyperbolyc rule of the lever) $m_{1} \sinh 2 \sqrt{-k} r_{1}=$ $m_{2} \sinh 2 \sqrt{-k} r_{2}$ and using the stereographic projection of a hyperbolic sphere on the Poincare disk, the center of mass for a system of two particles in $L_{\mathbb{R}}^{2}$ is given by

$$
m=m_{1} \cosh \left(2 \sqrt{-k} \ln \left(\frac{\left(R+w_{c}\right)\left(R-w_{1}\right)}{\left(R-w_{c}\right)\left(R+w_{1}\right)}\right)\right)+m_{2} \cosh \left(2 \sqrt{-k} \ln \left(\frac{\left(R-w_{c}\right)}{\left(R+w_{c}\right)} \frac{\left(R+w_{2}\right)}{\left(R-w_{2}\right)}\right)\right)
$$

Proof. Following the ideas of $[2,9]$, we can generalize, using the stereographic projection of a hyperbolic sphere on the Poincaré disk, the center of mass for a system of two particles in $L_{\mathbb{R}}^{2}$ our idea and so obtain the result. 
Remark 2. It has been established that if the particles have distinct masses, then the above definitions of the center of mass are not equivalent for hyperbolic spaces. Similarly, using the stereographic projection of a hyperbolic sphere on the Poincare disk, the three meanings for the center of mass (lever rule, collision point and center of steady rotation) are not equivalent. We consider that, from the physical point of view, the most appropriate definition is the definition present here, because it inherits two properties of the Euclidean center of mass (lever rule and collision point), while the relativistic definition only preserves one (conservation of angular momentum).

\section{Conclusions}

In the present work, an analytical formula is obtained that allows the exact calculation of the coordinates of the center of mass for a system of particles with positive masses located on a two-dimensional Riemannian manifold with constant and negative Gaussian curvature. The model of such a variety is taken as a model, Poincaré's disk $D^{2} R$, with the conformal metric resulting from the stereographic projection of the hyperbolic sphere $L^{2} R$. The formula obtained is derived using the hyperbolic lever law in this context, as one of the possibilities that is referenced in [4], and with it a previously obtained result is established more precisely that allows characterizing the relative equilibria for a 2-body problem on Poincaré's disk.

Author Contributions: P.P.O.P., A.M.M.R., and R.D.O.O. supervised the entire article. All authors performed the formal analysis, and participated in the writing and revising of the manuscript. All authors have read and agreed to the published version of the manuscript.

Funding: This research was funded by UNIVERSIDAD DE CARTAGENA grant number 062-2019.

Acknowledgments: The authors wishes to thank Joaquín Luna Torres and José Guadalupe Reyes Victoria, for their valuable suggestions.

Conflicts of Interest: The authors declare no conflict of interest.

\section{References}

1. Galperin, G.A. A concept of the mass center of a system of material points in the Constant Curvature Spaces. Commun. Math. Phys. 1993, 154, 63-84. [CrossRef]

2. Garcia-Naranjo, L.; Marrero J.C.; Perez-Chavela, E.; Rodriguez-Olmos, M. Classification and stability of relative equilibria for the two-body problem in the hyperbolic space of dimension 2. J. Differ. Equ. 2016, 260, 6375-6404. [CrossRef]

3. Diacu, F. The non-existence of centre-of-mass and linear-momentum integrals in the curved n-body problem. Lib. Math. 2012, 1, 25-37. [CrossRef]

4. Shchepetilov, A.V. Calculus and Mechanics on Two-Point Homogenous Riemannian Spaces; Springer: New York, NY, USA, 2006; Volume 707.

5. Diacu, F.; Perez-Chavela, E.; Reyes Victoria, J.G. An intrinsic approach in the curved n-body problem: The negative curvature case. J. Differ. Equ. 2012, 252, 4529-4562. [CrossRef]

6. Do Carmo, M. Differential Geometry of Curves and Surfaces; Prentice Hall: Upper Saddle River, NJ, USA, 1976.

7. Dubrovin, B.; Fomenko, A.; Novikov, P. Modern Geometry, Methods and Applications; Springer: New York, NY, USA, 1984, 1990; Volume I, II and III,

8. Ortega, P.P.; Ortiz, R.D.; Marin, A.M. Hyperbolic Center of Mass for a System of particles on the Poincaré Upper Half-Plane. Res. J. Appl. Sci. 2019, 14, 49-53.

9. Garcia-Naranjo, L.C. Some remarks about the centre of mass of two particles in spaces of constant curvature. Am. Inst. Math. Sci. 2020, 12, 435-446. [CrossRef] 